\title{
CLOSED-DIE FORGING OF GEAR LIKE ELEMENT
}

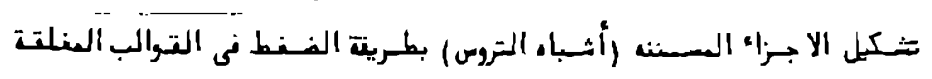

A. El-Domiaty, M. Shabara, M. Al-Ansary

Mech. Engg.Dept.

College of Engineering \&2Petroleum, Kuwait University, Kuwait.

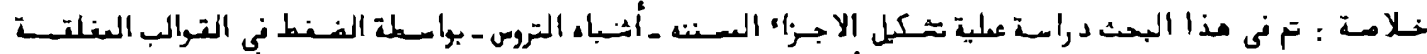

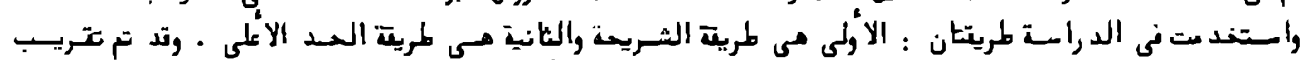

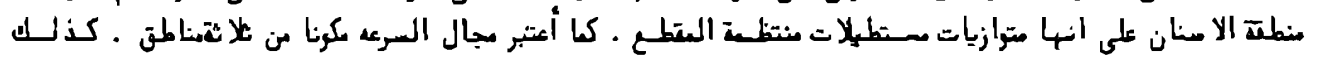

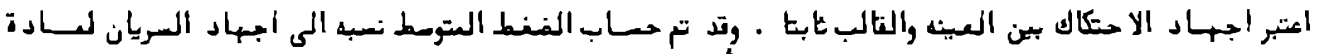

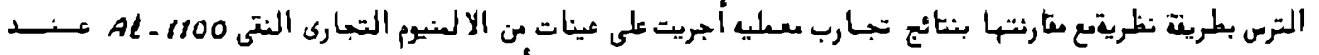

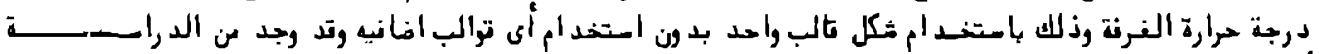

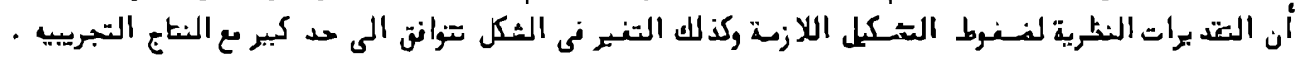

\section{ABSTRACT}

Closed die forging of spur gears is investigated using the slab method and the upper bound technique. The tooth regions are approximated by prismatic rectangular sections. The velocity field comprising three unit deformation regions is used. A constant frictional stress between workpiece and forging die is assumed.

The average punch pressure normalized by the flow stress of the gear material is determined theoretically and compared with experimental results. The experimental work is carried out on a commercial pure aluminum (A1 1100) at room temperature. The forging process is carried out using one die geometry without using any additional blocker (preform) dies.

The theoretical predictions of forging pressures and deformation configurations agree fairly well with the experimental results.

\section{INTRODUCTION}

With the use of closed-die forging, complex shapes and heavy reductions can be made within closer dimensional tolerances than are usually feasible with open dies. Closed-die forging is adaptable to high-volume production, improved structure, good mechanical properties and surface finish.

In closed-die forging, a material must satisfy two basic requirements: low flow stress and high forgability. The flow stress, which represents the resistance of the material to plastic deformation, must be low so that die pressures are kept within the capabilites of practical die materials. The ability of the material to deform without failure, i.e. its forgability, must allow for the desired amount of deformation. For a given material, both the flow stress and forgability are influenced by the metalurgical cheracteristics of the billet material and the forging parameters such as temperature, strain, strain rate and stresses.

In most practical closed-die forging operations, the temperature of the workpiece material becomes higher than that of the dies. Metal flow and die filling are largely determined by flow stress and forgeability of the billet material. Friction generally influences forgability, metal flow, pressure distribution, load and energy requirements. Therefore, the friction problem must be carefully dealt with by using an appropriate lubricant. 
The development of precision forging gear processes has got an area of increasing activity in recent years. Precision forged gears are made from billets having almost the exact volume of the material required for the final size of the gears. No allowance is needed for flash formation. Perform design problem in an axisymmetric disc forging where it is required to produce a uniformly deformed tat disc under the presence of friction at the die-workpiece interface had been carried out by Hwang and Kobayashi [1]. Yang and Kim [2] used the upper-bound method to determine the forging load and the deformation configurations during upset forging of elliptical disks. An analysis of comer filling characteristics in precision forging considering the effect of different workpiece geometries and lubrication wus carried out by Ibhandode and Dean[3]. The analysis showed that the velocity field which leads to the best load prediction is not that which most closely describes changes of geometry in a billet. Kim et al. [4] proposed some appropriate velocity fields for the upsetting analysis of three-dimensional forging of arbitranily-shaped prismatic blocks. The analysis showed a good agreement of forging load with their experimental results. Forging processes for heavy ingots by finite element method had been analyzed by Sun [5]. Large deformation behaviour of aluminum and low carbon steel shor anders loaded axially in a simple compression test without using any lubricant was examined by Gupta and Shah [6]. Their results revealed that the profile of a deforming specimen can be approximated by an arc of a circle only after the onset of folding.

Forging of spur gear forms and closed die forging of gear-like elements using the upper bound techrique have been anaiyzed $[7,8]$. The assumption of no axial velocity in the tooth region was shown to impose a very severe limitation on the validity of the analysis.

In the present work both the upper bound technique and the slab method have been used to analyze the closed-die forging of a spur gear. Numerical calculations have been done to study the effect of process and material variables on forging load estimation.

To determine the validity of the present analysis, an experimental program is carried out on commercially pure aluminum (AJ 1100) billets to forge a spur gear having twelve teeth.

\section{SLAB METHQD ANALYSIS}

A circular punch is used for forging cylindrical billets placed in a die having teeth cavities on its periphery. The punch compresses the billet axially and as a consequence the material flows outwards into the teeth spaces in the radial directions (see Fig. 1). In the initial stage of the forging process it is assumed that the billet aiready closely fits the die which has a diameter equal to the dedendum diameter and that the reduction in height allows the material to flow in the tooth cavities. The deformation pattern of the material of the billet which has a diameter equal to the root diameter of the gear is considered axisymmetric. The tooth formation is considered to take place as plane strain forging process. Integration for plane strain forging of teeth and axisymmetric forging of billet gives the complete solution of spur gear forging problem. 
The cylindrical and Cartesian coordinate systems are used to carry out the analysis, and are located as shown in Fig. 2. The axisymmetric state of stress of the billet and the state of the tooth are also shown in Fig. 2.

By considering the equilibrium of forces in the $x$-direction, the plane strain condition, and the Von Mises yield criterion, the following differential equation is obtained.

$$
\frac{\mathrm{d} \sigma_{\mathrm{x}}}{\mathrm{dx}}+2 \mu\left(\frac{1}{\mathrm{w}}+\frac{1}{\mathrm{~h}}\right) \sigma_{\mathrm{s}}=\left(\frac{4 \mu}{\sqrt{3} \mathrm{~h}}+\frac{2 \mu}{\sqrt{3} \mathrm{w}}\right) \bar{\sigma}
$$

If $\bar{\sigma}$ is not a function of $x$, Eqn. 1 becomes

$$
\frac{\mathrm{d} \sigma_{x}}{\mathrm{dx}}+\mathrm{c}_{1} \sigma_{\mathrm{x}}=\mathrm{c}_{2}
$$

where $\quad c_{1}=2 \mu\left(\frac{1}{w}+\frac{1}{h}\right)$

and

$$
c_{2}=\left(\frac{4 \mu}{\sqrt{3} h}+\frac{2 \mu}{\sqrt{3} w}\right) \bar{\sigma}
$$

The solution of Eqn. 2 is:

$$
\sigma_{z}=\left(\frac{c_{2}}{c_{1}}\right)+D \mathrm{e}^{-c_{1} x}
$$

where $D$ is an integration constant which can be determined from the boundary conditions

$$
\sigma_{x}=0 \quad \text { at } \mathrm{x}=\ell \quad \text { (before complete filling) }
$$

Solving for $\mathrm{D}$, we find:

$$
D=-\left(\frac{c_{2}}{c_{1}}\right) e^{c_{1} l}
$$

From Eqns. 4 and $S$, the stress $\sigma_{x}$ is

$$
\sigma_{x}=\frac{c_{2}}{c_{1}}\left[1-e^{c_{1}(c-x)}\right]
$$

The axial stress, $\sigma_{y}$, can then be expressed as:

$$
\sigma_{y}=\frac{2}{\sqrt{3}} \bar{\sigma}\left\{\mathrm{c}\left[1-\mathrm{e}^{c_{1}(t-\mathrm{x})}\right]-1\right\}
$$


where $\quad c=\left(\frac{1}{h}+\frac{1}{2 w}\right) /\left(\frac{1}{h}+\frac{1}{w}\right)$

The load required to forge the teeth at any stage of deformation can be obtained by equating the external load to the internal resistance, that is:

$$
(\text { Load })_{\text {teeth }}=P_{\mathrm{av}}(w \ell) N=N \int_{0}^{l} \sigma_{y} w d x
$$

where $\mathrm{N}=$ number of teeth

Hence, the normalized average pressure required to forge the material in the tooth region becomes:

$$
\left[\frac{P_{w}}{\bar{\sigma}}\right]_{\text {wath }}=\frac{2}{\sqrt{3}}\left[\left(c-1+\frac{c}{C_{1}}\right)\left(1-e^{C_{1} \ell}\right)\right]
$$

For the axisymmetric part of the gear which is bound by the circle of radius $r_{2}$, the force equilibrium in the $r$-direction gives:

$$
\frac{\mathrm{d} \sigma_{\mathrm{r}}}{\mathrm{dr}}+\frac{2 \mu}{\mathrm{h}} \sigma_{\mathrm{r}}=\frac{2 \mu}{\mathrm{h}} \bar{\sigma}
$$

The solution of Eqn. 11 is:

$$
\sigma_{1}=\mathrm{e}^{-2 \mu \mathrm{r} / \mathrm{h}}\left[\bar{\sigma} \mathrm{e}^{2 \mu \mathrm{r} / \mathrm{h}}+\mathrm{Q}\right]
$$

where $Q$ is a constant which can be determined from the boundary condition

$$
\left[\sigma_{r}\right]_{1=r_{2}}=\left[\sigma_{x}\right]_{x=0}
$$

Then

$$
Q=\left[\frac{c_{2}}{c_{1}}\left(1-e^{c_{1} \ell}\right)-\bar{\sigma}\right] e^{D_{1}}
$$

where

$$
D_{1}=2 \mu r_{2} / h
$$

From Eqns. 12 and 13, the stress in the r-direction becomes:

$$
\sigma_{t}=e^{-2 \mu / h}\left\{\bar{\sigma} e^{1 \mu t / h}+\left[\frac{c_{2}}{c_{1}}\left(1-e^{c_{1}}\right)-\bar{\sigma}\right] e^{U_{1}}\right\}
$$


Using the axisymmetric condition $\sigma_{\mathrm{r}}=\sigma_{\theta}$ in Von-Mises yielding criterion leads to:

$$
\sigma_{2}=\sigma_{r}-\bar{\sigma}
$$

From Eqns. 14 and 15,

$$
\sigma_{\mathrm{x}}=\mathrm{e}^{\left(\mathrm{D}_{1}-\mathrm{D}_{2}\right)}\left[\frac{\mathrm{C}_{2}}{\mathrm{C}_{1}}\left(1-\mathrm{e}^{\mathrm{C}_{1} l}\right)-\bar{\sigma}\right]
$$

The forging load for the axisymmetric part of the gear is obtained as follows:

$$
\begin{aligned}
& F_{u x}=\int_{0}^{r_{z}} 2 \pi r d r \sigma_{r} \\
\text { Also, } \quad F_{2 x} & =P_{w v} \pi r_{2}^{2}
\end{aligned}
$$

By substituting Eqn. 16 into Eqn. 17-a, equating to Eqn. 17-b, and integrating, the normalized average pressure required to forge the material in the axisymmetric part of the gear becomes:

$$
\left[\frac{\mathrm{P}_{\mathrm{av}}}{\bar{\sigma}}\right]_{\mathrm{u}}=\frac{2}{\mathrm{r}_{2}^{2}}\left[\frac{2}{\sqrt{3}} \mathrm{c}\left(1-\mathrm{e}^{\mathrm{C}_{\mathrm{l}} \ell}\right)-\mathrm{l}\right] \mathrm{e}^{\mathrm{D}_{1} I}
$$

where

$$
\begin{gathered}
I=-e^{D_{2} r_{2}}\left[\frac{r_{1}}{D_{2}}+\frac{1}{D_{2}^{2}}\right]+\frac{1}{D_{2}^{2}} \\
D_{2}=2 \mu / h
\end{gathered}
$$

The constants $C_{1} c_{1}$, and $D_{1}$ have been previously defined

Eqn. 18 gives the normalized average pressure required to forge the material in the axisymmetric part of the gear.

The tooth length at any time during the forging process can be calculated using the constant volume concept as follows:

$$
\pi r_{2}^{2} h_{0}=\pi r_{2}^{2} h+N w \ell h
$$

Hence, $\quad \ell=r_{1}\left[\frac{h_{0}}{h}-1\right]$ 
M. 38 A. El-Danlaty, M. Shabara, M. El-Ansary

The total pressure needed to form the gear is assumed to be the sum of two portions. The first portion is the pressure needed to deform the billet multiplied by an area factor $f_{1}$. The second portion is the pressure needed to form the teeth multiplied by another area factor

$f_{2}$. The area factors are: $f_{1}=\frac{A_{\alpha x}}{A_{1}}$ and $f_{2}=\frac{A_{\text {ounh }}}{A_{1}}$ where $A_{1}=A_{\alpha}+A_{\text {conte }}$.

Using Eqs. 18 and 10 , the total average pressure $\left(P_{2 y}\right)$ normalized by the flow stress of the material $\bar{\sigma}$ becomes

$$
\left[\frac{P_{w}}{\bar{\sigma}}\right]_{\text {wav }}=\left[\frac{P_{w}}{\bar{\sigma}}\right] f_{1}+\left[\frac{P_{w}}{\bar{\sigma}}\right] f_{2}
$$

where $A_{\text {, }}$ is the total cross sectional area of the gear

\section{THE UPPER BOUND ANALYSIS}

A cylindrical billet for forging a gear with $\mathrm{N}$ teeth can be divided into $2 \mathrm{~N}$ deformation units. One of these deformation units (as shown in Fig. 3) is bounded by the contour OBCDEMO. The planes of symmetry OB and OME make an angle $a$ between them and separate the deformation unit OBCDEMO from the adjacent units. No metal can cross or shear along a plane of symmetry. Therefore, the material in the unit, displaced by downward punch displacement is forced to flow to form half a tooth. Each deformation unit is divided into three regions of deformation designated (I), (II) and (III) as shown in Fig. 3.

\section{Velocity and Strain Rate Fields}

Radial flow cannot take place in region I because of the constraint imposed by the die wall BC (see Fig. 3). Therefore material flows tangentially from region I to region II as the punch moves downward. As the deformation progresses, the material flows to the tooth in region IIl. The velocity fields in each deformation region are discussed below.

\section{Region I:}

As mentioned above, there is no radial velocity, $u$, in this region and the axial velocity, $w$, is given by:

$$
\mathbf{w}=-\mathbf{z} U / \mathbf{t}
$$

Where
$z=$ the height of the tooth
$\mathrm{U}=$ punch downward velocity
$\mathrm{t}=$ the billet thickness at any time during the deformation process 
Using the continuity equation in the cylindrical coordinates for the flow of the material, the velocity component in the tangential direction can be written as:

$$
v=\frac{\cdot U(\alpha-\theta) r^{*}}{t}
$$

The strain rates corresponding to the velocity components are:

$$
\begin{aligned}
& \dot{\varepsilon}_{\mathrm{r}}=\dot{\gamma}_{\mathrm{r} \theta}=\dot{\gamma}_{\mathrm{zr}}=0 \\
& \dot{\varepsilon}_{\theta}=-\dot{\varepsilon}_{\mathrm{z}}=\mathrm{U} / \mathrm{t}
\end{aligned}
$$

The effective strain rate in this region becomes:

$$
\begin{aligned}
\dot{\bar{\varepsilon}} & =\sqrt{\left(\dot{\varepsilon}_{r}^{2}+\dot{\varepsilon}_{0}^{2}+\dot{\varepsilon}_{z}^{2}+0.5\left(\dot{\gamma}_{r \theta}^{2}+\dot{\gamma}_{\theta z}^{2}+\dot{\gamma}_{z}^{2}\right)\right\}} \\
& =\sqrt{2} \mathrm{U} / \mathrm{t}
\end{aligned}
$$

The calculation of the loads needed to cause plastic fow of the metal is ofen difficult, if not impossible. Exact solutions require that both stress equilibrium and geometrically selfconsistent pattern of flow are satisfied simultaneously everywhere throughout the deforming body. The upper-bound analysis offers a technique which predicts a load that is at least equal to or greater than the exact load needed to cause piastic flow. Using the upper-bound analysis, the external forces are calculated by equating the external work with the internal energy consumption or dissipation. The rate of energy dissipation $\dot{E}$ can be calculated based on the assumed velocity fields, as the sum of the energy rate for plastic deformation, $\dot{\mathrm{E}}_{p}$, the rate of energy dissipation due to the velocity discontinuity, $\dot{\mathrm{E}}_{\mathrm{f}}$, and the rate of energy dissipation due to the friction between the defomed material and the forming dies, $\dot{E}_{f}$. That is:

$$
\begin{aligned}
& \dot{E}=\dot{E}_{p}+\dot{E}_{d}+\dot{E}_{l} \\
& =\int_{v} \bar{\sigma} \bar{\varepsilon} d v+\int_{a} \tau v^{*} \mid d s+\int m \tau v_{v i t} d s
\end{aligned}
$$

The energy rates $\dot{E}_{p}, \dot{E}_{d}$, and $\dot{E}_{f}$ in region I are calculated as follows:

(a) Assuming that the material behaves according to Von Mises Yield criterion, then the energy rate for plastic deformation in Region I is given by

$$
\dot{\mathrm{E}}_{\mathrm{p} 1}=\sqrt{\frac{2}{3}} \bar{\sigma} \iiint \dot{\bar{\varepsilon}} \mathrm{r} \mathrm{dr} \mathrm{d} \theta \mathrm{dr}=\frac{\mathrm{U}}{\sqrt{3}} \bar{\sigma}(\alpha-\beta) \mathrm{r}_{2}^{2}
$$


(b) The fiction energy dissipated during the forging process is the sum of the friction

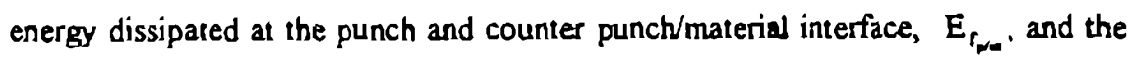
friction energy dissipated at the die wall/material interface, $\mathrm{BC}, \mathrm{E}_{\mathrm{r}_{\mathrm{u}} \cdot \mathrm{e}}$. The rates of these energies, in region $I$, are expressed as:

$$
\left.E_{\mathrm{r}_{\mathrm{p} / \alpha}}\right|_{\mathrm{r}}=\frac{\mathrm{m}}{\sqrt{3}} \bar{\sigma} \iint \mathrm{v} \mathrm{r} d \theta \mathrm{dr}=\frac{2 \mathrm{~m} U \mathrm{r}_{2}^{\mathrm{j}} \bar{\sigma}}{3 \sqrt{3} \mathrm{l}}(\alpha-\beta)^{2}
$$

where $m$ is a friction factor, and

$$
E_{r_{1 /-}} \mid r=\frac{m \bar{\sigma}}{\sqrt{3}} \iint \Delta v r_{2} d \theta d z=\frac{m r_{2} \bar{\sigma} U}{2 \sqrt{3} t} \int_{\beta}^{a} l d \theta
$$

where $\Delta v$ is the relative sliding velocity which is the resultant of axial and circumferential velocities at the interface, i.e.

$$
\text { and } \begin{aligned}
\Delta v= & \sqrt{\left.\left[u_{\theta}^{2}+u_{z}^{2}\right]\right|_{r=r_{2}}}=\frac{U}{t} \sqrt{\left[r_{2}^{2}(\alpha-\theta)^{2}+z^{2}\right]} \\
& t \sqrt{r_{2}^{2}(\alpha-\theta)^{2}+t^{2}}+r_{2}^{2}(\alpha-\theta)^{2} \ln \left[t+\sqrt{r_{2}^{2}(\alpha-\theta)^{2}+t^{2}}\right] \\
& -r_{2}^{2}(\alpha-\theta)^{2} \ln \left[r_{2}(\alpha-\theta)\right]
\end{aligned}
$$

The value of $\int_{\theta}^{a} I \mathrm{~d} \theta$ can be obtained by numerical integration. ,

(c) The energy dissipation due to the velocity discontinuity in region I is zero.

\section{Region II}

Region II is defined by the boundary OCM in Fig. 3.

The tangential velocity varies from maximum at the boundary $\mathrm{OC}, \theta=\beta$, to zero at the boundary $\operatorname{OM} \theta=0$. Using the incompressibility condition along with the boundary conditions, the velocity components in region II can be written as:

$$
\begin{aligned}
& u=\frac{U \alpha r}{2 t \beta} \\
& v=-\frac{U(\alpha-\beta) \theta r}{t \beta}
\end{aligned}
$$




$$
w=-\frac{U}{t} z
$$

The strain rate field is determined from the velocity field (Eqns. 30, 31 and 32) as:

$$
\begin{aligned}
& \dot{\varepsilon}_{\mathrm{r}}=U \alpha /(2 \mathrm{t} \beta) \\
& \dot{\varepsilon}_{\theta}=U(2 \beta-\alpha) /(2 \mathrm{t} \beta) \\
& \dot{\varepsilon}_{2}=-U / \mathrm{t} \\
& \dot{\gamma}_{t \theta}=\dot{\gamma}_{\theta 2}=\dot{\gamma}_{z}=0
\end{aligned}
$$

The effective strain rate in region $I I$ is determined from Eqns. 25 and 33 as:

$$
\dot{\bar{\varepsilon}}_{2}=U \sqrt{\left(\alpha^{2}-2 \alpha \beta+4 \beta^{2}\right)} /(\sqrt{2 \mathrm{t}} \beta)
$$

Substituting the effective strain rate $\dot{\bar{\varepsilon}}_{2}$ (Eqn. 34) into Eqn. 26, the energy for plastic deformation in region 11 is:

$$
\begin{aligned}
E_{p l l} & =\frac{\sqrt{2}}{3} \bar{\sigma} \iiint \dot{\bar{\varepsilon}}_{2} \mathrm{r} d r \mathrm{~d} \theta \mathrm{d} z \\
& =2 U \sqrt{3} \bar{\sigma} \mathrm{r}_{2}^{2} \sqrt{\alpha^{2}-2 \alpha \beta+4 \beta^{2}}
\end{aligned}
$$

The frictional energy dissipated at the punch/material interface is due to the sliding velocity $v_{f}$ which is the resultant of the radial and the tangential velocities at the interface. This is given by:

$$
\begin{aligned}
v_{\mathrm{f}} & =\left|\sqrt{\left(\mathrm{v}_{\theta}^{2}+\mathrm{v}_{\mathrm{l}}^{2}\right)}\right|_{\mathrm{z}=\mathrm{t}} \\
& =\frac{U_{\mathrm{r}}}{2 \mathrm{t} \beta} \sqrt{\alpha^{2}+4(\alpha-\beta)^{2} \theta^{2}}
\end{aligned}
$$

Therefore, the frictional energy dissipated is given by:

$$
\begin{aligned}
\mathrm{E}_{\mathrm{r} \theta}= & \frac{2 \mathrm{~m} \vec{\sigma}}{\sqrt{3}} \iint_{\theta} \mathrm{v}_{\tau} \mathrm{r} \mathrm{d} \theta \mathrm{dr} \\
& =\frac{2 \mathrm{~m} \bar{\sigma}}{\sqrt{3}} \int_{0}^{g_{1}} \int_{0}^{\left(r_{2} \cos \mu_{\cos \theta)}\right.} \frac{U_{r}}{2 \mathrm{t} \beta} \sqrt{\alpha^{2}+4(\alpha-\beta)^{2} \theta^{2}} \mathrm{rdr} d \theta
\end{aligned}
$$




$$
=\frac{\mathrm{m} \bar{\sigma} U \mathrm{r}_{2}^{2} \cos ^{2} \beta}{2 \sqrt{3} \mathrm{t} \beta}
$$

The integral $I_{2}=\int_{0}^{\theta} \sqrt{4(\alpha-\beta)^{2} \theta^{2}+\alpha^{2}} \sec ^{2} \theta d \theta$ is to be evaluated numerically.

The shear energy due to velocity discontinuity at the surface $O C$ is due to the difference in radial velocities in regions $I$ and $I I$. This is:

$$
v_{d}=\frac{U \alpha}{2 t \beta} r
$$

Therefore the shear power dissipated at surface $O C$ is given by:

$$
E_{d}=\frac{\bar{\sigma}}{\sqrt{3}} \iint v_{d} d r d z=\frac{U \bar{\sigma} \alpha r_{2}^{2}}{4 \sqrt{3} \beta}
$$

\section{Region III}

This region is defined by the boundary CDEM in Fig. 3. The tangential velocity in this region is assumed to be zero. The admissible velocity field is found by considering the velocity field at any point on the surface of the velocity discontinuity $C M$ such as point $q$, where $r=r_{2}$.

From Eqns. 30 and 31 , the velocity field at point $q$ is given by:

$$
\text { and } \quad \begin{aligned}
\mathrm{u}_{\mathrm{q}} & =\frac{\mathrm{U} \alpha \mathrm{r}_{2}}{2 \mathrm{t} \beta} \\
\quad \mathrm{v}_{\mathrm{q}} & =\frac{U \mathrm{r}_{2}(\alpha-\beta)}{\mathrm{t}}
\end{aligned}
$$

The incompressibility condition in cylindrical coordinates is expressed by zero volumetric strain rates, i.e.,

$$
\frac{\partial u}{\partial r}+\frac{u}{r}+\frac{1}{r} \frac{\partial v}{\partial \theta}+\frac{\partial w}{\partial z}=0
$$

The radial velocity in region III is determined by using Eqn. 41 as follows:

$$
u=\left[\frac{r}{2 t}+\frac{k}{r}\right] U
$$


At point $q, r=r_{2}, u=u_{q}$ and the constant $k$ becomes:

$$
\text { - } k=r_{2} \frac{u_{q}}{U}-\frac{r_{2}^{2}}{2 t}
$$

The velocity discontinuity at $\mathrm{CM}$ is due to the tangential velocity at the surface in region II relative to that at the surface in region III. The velocity discontiunity is given by:

$$
\mathrm{v}_{\mathrm{d}}=-\mathrm{U}\left[\frac{\mathrm{r}_{2}(\alpha-\beta) \theta}{\mathrm{t} \beta}+\frac{\alpha \mathrm{r}_{2}}{2 \mathrm{t} \beta} \tan \theta\right]
$$

Hence the strain rate components are:

$$
\begin{aligned}
& \dot{\varepsilon}_{r}=U\left[\frac{1}{2 t}-\frac{k}{r^{2}}\right] \\
& \dot{\varepsilon}_{\theta}=U\left[\frac{1}{2 t}+\frac{k}{r^{2}}\right] \\
& \dot{\varepsilon}_{2}=-\frac{U}{t} \\
& \dot{r}_{t \theta}=\dot{\varepsilon}_{\theta z}=\dot{\varepsilon}_{\mathrm{st}}=0
\end{aligned}
$$

The effective strain rate becomes:

$$
\dot{\bar{\varepsilon}}_{3}=U\left\{\frac{3}{2 t^{2}}+\frac{2 k^{2}}{r^{4}}\right\}^{0.5}
$$

The plastic deformation energy in region III is given by

$$
\begin{aligned}
E_{\text {pitI }} & =\sqrt{\frac{2}{3}} \bar{\sigma} \iint_{s} \dot{\bar{\varepsilon}}\left(r_{2} \beta\right) d r d z \\
& =\sqrt{\frac{2}{3}} r_{2} \beta t U \bar{\sigma} \int_{r_{1}}^{r_{3}}\left\{\frac{3}{2 t^{2}}+\frac{2 k^{2}}{r^{4}}\right\}^{0 s} d r
\end{aligned}
$$

The frictional energy at the punch/material interface in region III is given by:

$$
\left.E_{r_{p, m}}\right|_{\mathrm{m}}=\frac{2 m r^{2}}{\sqrt{3}} \beta \bar{\sigma}\left[\frac{\left(r_{3}^{2}-r_{1}^{2}\right)}{4 t}+k n\left(\frac{r_{3}}{r_{2}}\right)\right]
$$


The frictional energy dissipated at the die/material interface $C D$ is due to the resultant velocity along $C D$ which is given by:

$$
v=\frac{U}{t}\left\{\left(\frac{r}{2}+\frac{t k}{r}\right)^{2}+z^{2}\right\}^{a s}
$$

Therefore the frictional energy dissipated at the die/material interface $C D$ is given by

$$
E_{r_{\text {s/m }}} / \text { IIt }=\frac{m \bar{s}}{\sqrt{3}} \iint_{z} v d r d z
$$

where

$v$ is the sliding velocity given by Eqn. 49 .

The shear energy dissipated at the die/material interface $C D$ is due to the resultant velocity along $C D$ which is given by:

$$
\begin{aligned}
E_{d_{\mathrm{m}}} & =\frac{\bar{\sigma}}{\sqrt{3}} \iint_{\theta} v_{\mathrm{z}} r_{2} d \theta d z \\
& =-\frac{1}{2 \sqrt{3}} r_{2}+\bar{\sigma}\left\{\frac{r_{2}(\alpha-\beta)}{\mathrm{t}} \beta-\frac{\alpha \mathrm{r}^{2}}{\mathrm{t} \beta} \ell_{n} \cos \beta\right\}
\end{aligned}
$$

where $v_{d}$ is the velocity discontinuity given by Eqn. 44 .

The total energy dissipation for the complete region is obtained by summing up all the energy components in the three deformation regions, i.e.,

where

$$
\dot{\mathrm{E}}=\dot{\mathrm{E}}_{1}+\dot{\mathrm{E}}_{11}+\dot{\mathrm{E}}_{11}
$$

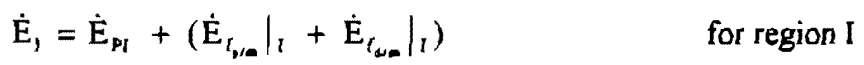

$$
\begin{aligned}
& \dot{\mathrm{E}}_{\mathrm{n}}=\dot{\mathrm{E}}_{p_{\mathrm{g}}}+\dot{\mathrm{E}}_{i_{\mathrm{q}}}+\dot{\mathrm{E}}_{d_{\mathrm{q}}} \quad \text { for region II } \\
& =\dot{E}_{p \mid 11}+\left(\dot{E}_{t p / m}\left|m+\dot{E}_{r d / m}\right|_{m}\right)+\dot{E}_{d I n} \text { for region III }
\end{aligned}
$$

For the complete gear, the energy required for forging is:

$$
\dot{\mathrm{E}}_{1}=2 \mathrm{~N} \dot{\mathrm{E}}
$$

The punch moves down with a velocity $U$ applying a force $P$. Hence, the external power consumed in the forging process $=P U=P_{a v} \times A \times U=\dot{E}$, 
where:

$A=$ area of the deformed gear normal to the average applied pressure $\left[\mathrm{P}_{\mathrm{av}}\right]$ by the punch.

By equating the extemal power to the internal power required for forging, we obtain:

$$
\begin{aligned}
& \dot{\mathrm{E}}_{\mathrm{t}}=2 \mathrm{NE}=\mathrm{P}_{د v} A \mathrm{U} \\
& \mathrm{P}_{\Delta v}=\dot{\mathrm{E}}_{\mathrm{t}} / \mathrm{AU} \\
& {\left[\frac{\mathrm{P}_{a v}}{\bar{\sigma}}\right]=\frac{\dot{\mathrm{E}}_{1} / \bar{\sigma}}{\mathrm{AU}}}
\end{aligned}
$$

\section{EXPERIMENTAL WORK}

Cylindrical billets of $32-\mathrm{mm}$ diameter and 14-mm thickness made of commercially pure aluminum were closed-forged in a special die which has twelve cavities which form the teeth of the gear. The specially designed die and the associated tooling are shown in Fig. 4. Prior to the forging process, compression tests were carried out on the cylindrical specimens to obtain the flow stress of the material at room temperature. During the forging process, the load-punch travel curve was recorded by the testing machine. Both the compression tests and the forging process were carried out at the same temperature and head speed of the testing machine.

\section{RESULTS AND DISCUSSION}

Fig. 5 shows the cylindrical billet and the formed gear. The calculated progressive increase of tooth length . Eqn. 20, is plotted against the reduction in billet height $h$ as shown in Fig. 6 . The numerical values of the relative average punch pressure, $\mathrm{P}_{\mathrm{av}} / \bar{\sigma}$, are determined for twelve teeth gear with root diameter of $32 \mathrm{~mm}$ by using the slab method, Eqn. 21 , and the upper bound technique, Eqn. 53 and are shown in Fig. 7. These results are obtained for a constant value of the coefficient of friction $\mu=0.3$. It is clearly demonstrated in Fig. 7 that the relative average punch pressure, $P_{a v} / \bar{\sigma}$ is increasing in an exponential form as the reduction in height of the billet increases.

The experimental results of the closed die forging of the gear element are obtained in the form of load versus reduction in height as shown in Fig. 8. These results are transferred to be in the form of relative average pressure versus reduction in height (see Fig. 9) to make possible the comparison between the theoretical results, by both slab and upper bound method, and the experimental results. In order to transfer the experimental results, the flow stress of the material must be known as a function of the reduction in height. This function is determined by simple compression test at room temperature and the results are 
shown in Fig. 10 and Fig. 11. From the results in Fig. 9 and Fig. 11, the experimental values of $\left(P_{\mathrm{av}} / \bar{\sigma}\right)$ as a function of reduction in height are obtained and shown in Fig. 12.

A comparison between the experimental and the theoretical result are shown in Fig. 13 . It $^{\circ}$ is possible to see from the results shown in Fig. 13 that the slab method over- estimates the values of $\left(\mathrm{P}_{\mathrm{av}} / \bar{\sigma}\right)$ for small values of reduction in height while it gives very close values to the experimental values for high values of reduction in height. Also from the results shown in Fig. 13 it is shown that the upper bound technique gives very close values for $\left(\mathrm{P}_{\mathrm{av}} / \bar{\sigma}\right)$ for high values reduction in height. This means that the upper bound technique underestimates the values of $\left(\mathrm{P}_{\mathrm{av}} / \bar{\sigma}\right)$ before the complete filling of the die corners, while it gives very close values of $\left(\boldsymbol{P}_{\text {av }} / \bar{\sigma}\right)$ during the filling process.

\section{CONCLUSION}

The theoretical predictions for the relative average pressure $\left(\mathrm{P}_{\mathrm{av}} / \bar{\sigma}\right)$ needed for forging of a spur gear by using the slab and upper bound techniques are introduced. These predictions are in good agreement with the experimental results which are obtained for forging a twelve-teeth aluminum gear.

\section{REFERENCES}

[1] S.M. Hwang and S. Kobayashi, Perform design in disk forging, Int. J. Mech. Des. Res. Vol. 26, No. 3. pp. 231-243, 1986.

[2] D.Y. Yang and J.H. Kim, "An analysis for three-dimensional upset forging of elliptical disks", Int. J. Mech. Tool Des. Res. Vol. 26 No., pp. 147-156, 1986.

[3] A. O. A. Ibhandode and T. A. Dean, "Corner filling characteristics in precision forging", Int. J. Mech. Tools Manufact., Vol. 28, No. 2, pp. 103-122, 1988.

[4] J.H. Kim, D.Y. Yang and M. U. Kim, "Analysis of three-dimensional upset forging of arbitrarily-shaped prismatic blocks", Int. J. Mech. Tools Manufact., Vol. 27, No. 3, pp. 311-323, 1987.

[5] J.X. Sun," Analysis of special forging processes for heavy ingots by finite element method", Int. J., Mech. Tolls Manufact. Vol. 28, No. 2, pp. 113-179, 1988.

[6] N.K. Gupta and C.B. Shah, "Barreling of short cylinder in compression", Int. of Mech. Tool Des. Res. Vol. 20, No. 2, pp. 137-146, 1986

[7] N.A. Abdul and T.A. Dean, "An Analysis of the forging of spur gear forms", Int. J. Mech. Tool Des. Res. Vol. 26, No. 2, pp. 113-123, 1986

[8] O.P. Grover and L. Juneja "Analysis of closed-die forging of gear-like element: ", Advanced Technology of Plasticity", Vol. II, The Japan Society for Technology of Plasticity, pp. 888-893, 1984. 


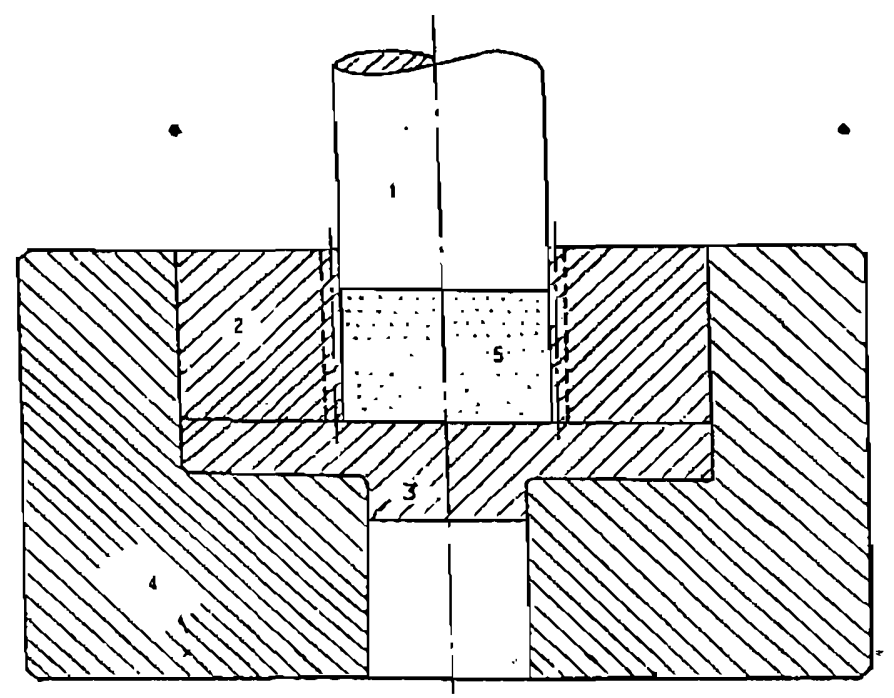

1- Punch, 2- Die, 3- Dle Support, 4- Contalner

5- Yorkplece

F16. 1: THE FORHIHG TOOLS

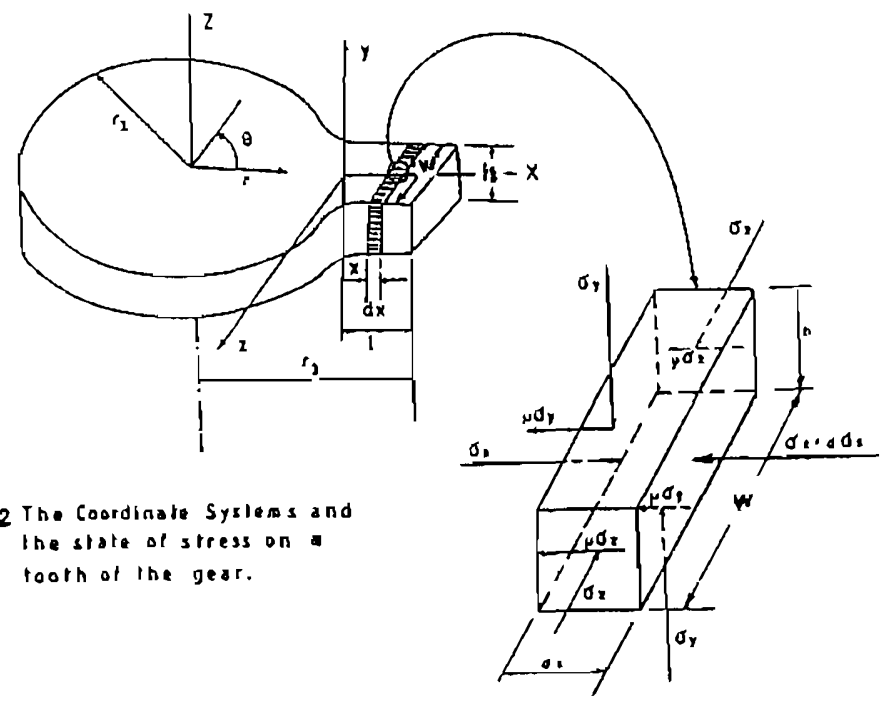

fig. J The three deloenalion regions forming one delormalion unit.

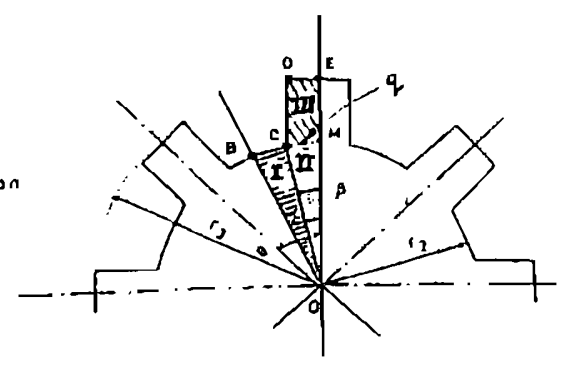




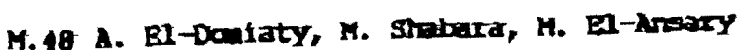

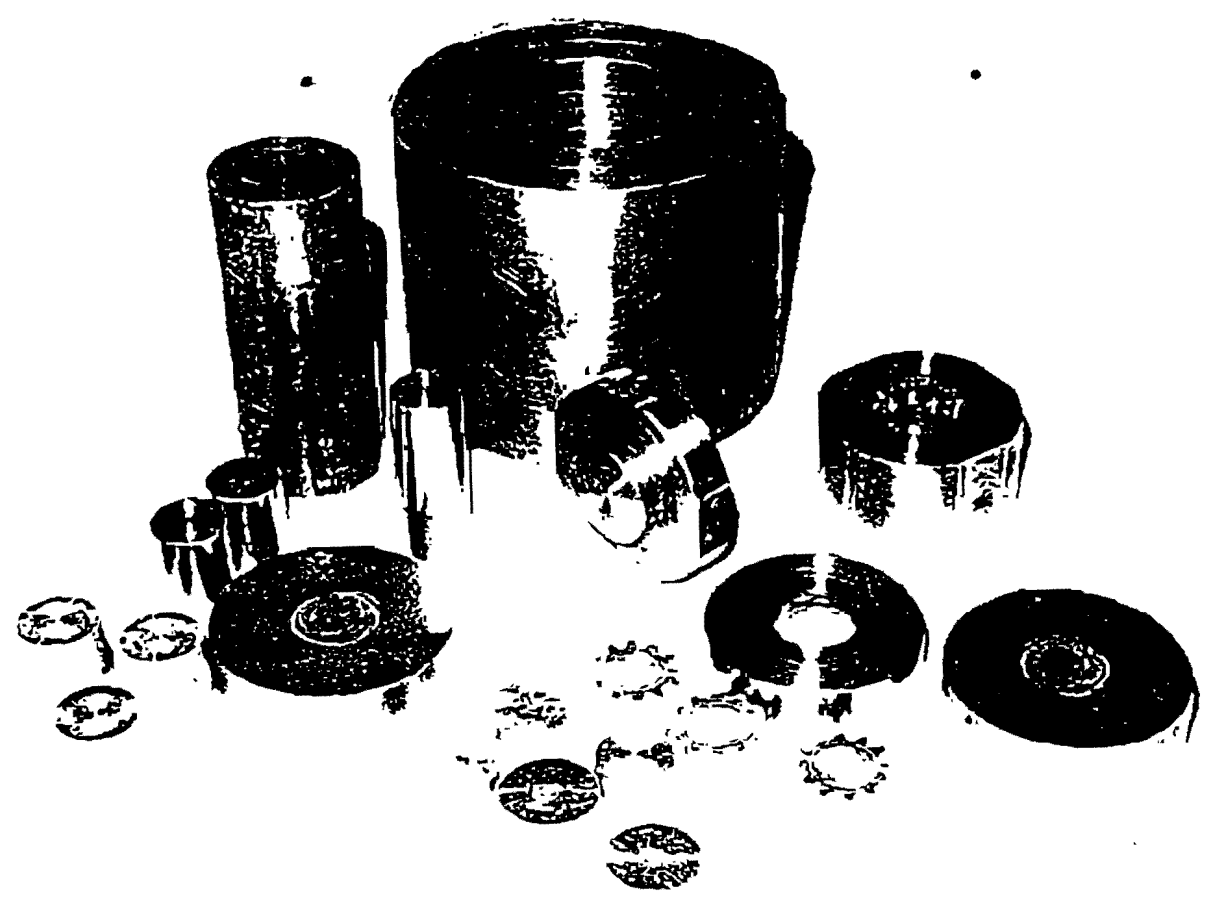

Fig. 4: The closed die forging tools.
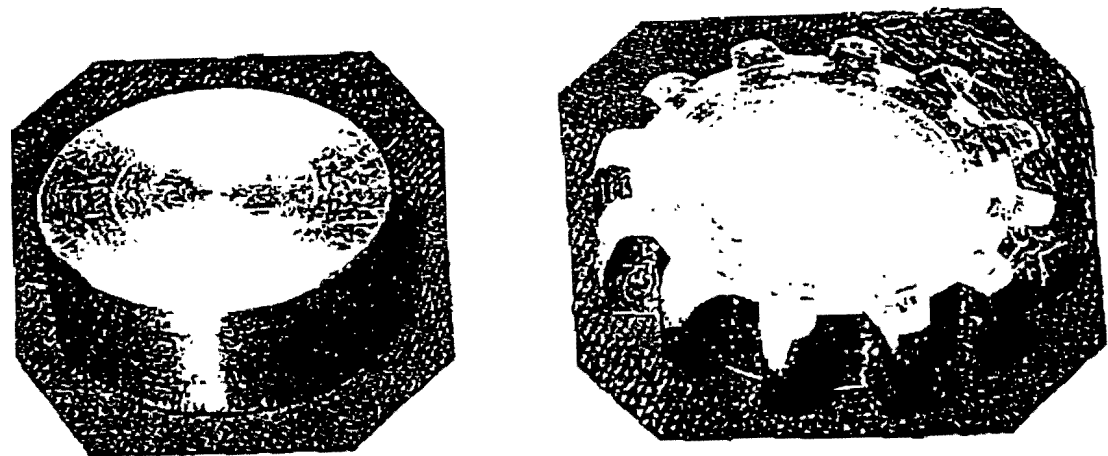

fig. 5 : The aluminum billet in initial and final form after forging. 


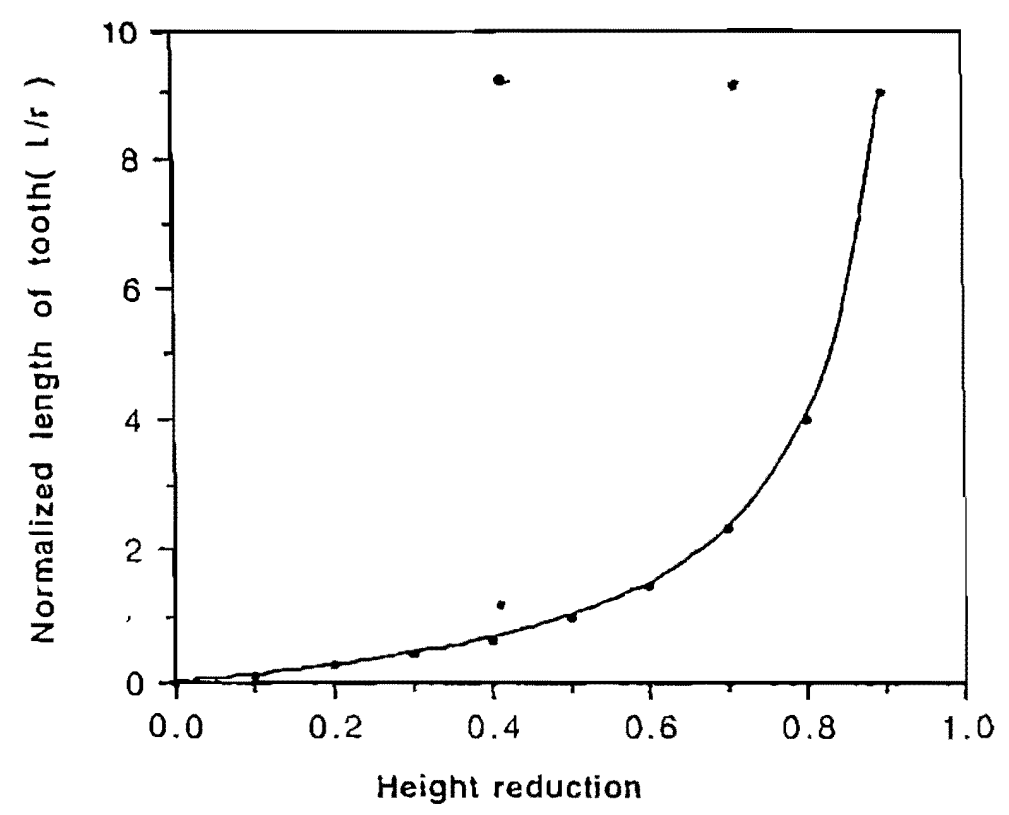

Fig.(6) Tooth length as a function of height reduction

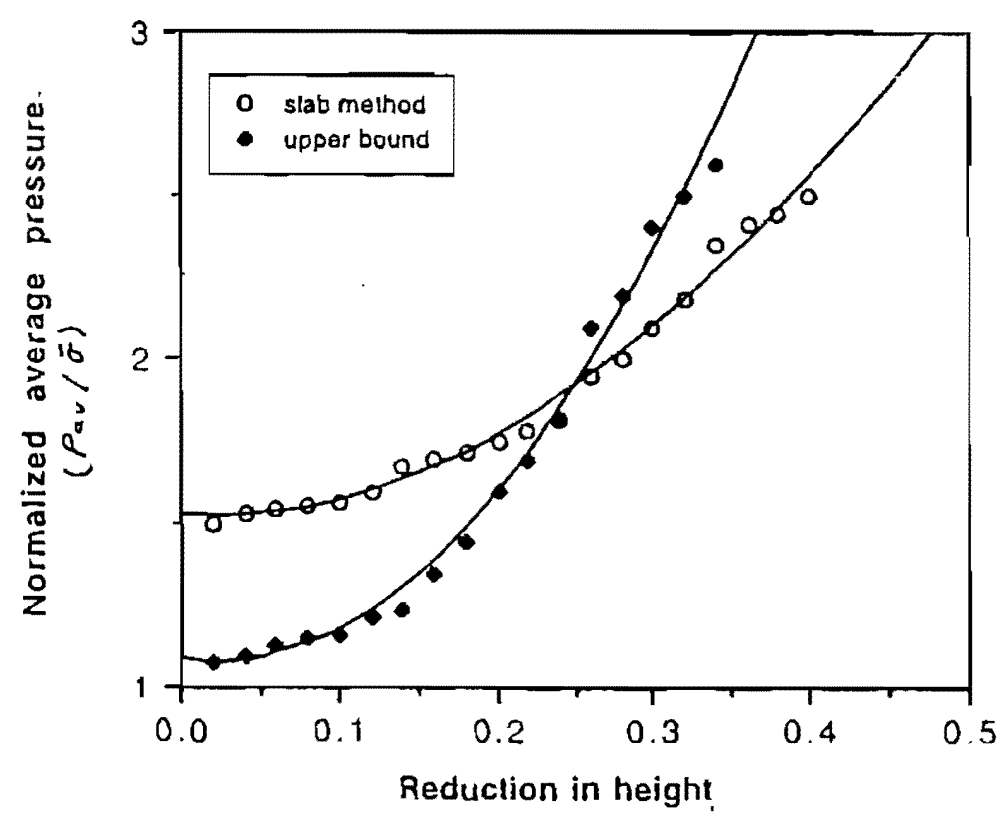

Fig.(7) Comparison between the slab and the upper bound methods. 
M.50 A. El-Domlaty, M. Shabara, M. E1-Ansary

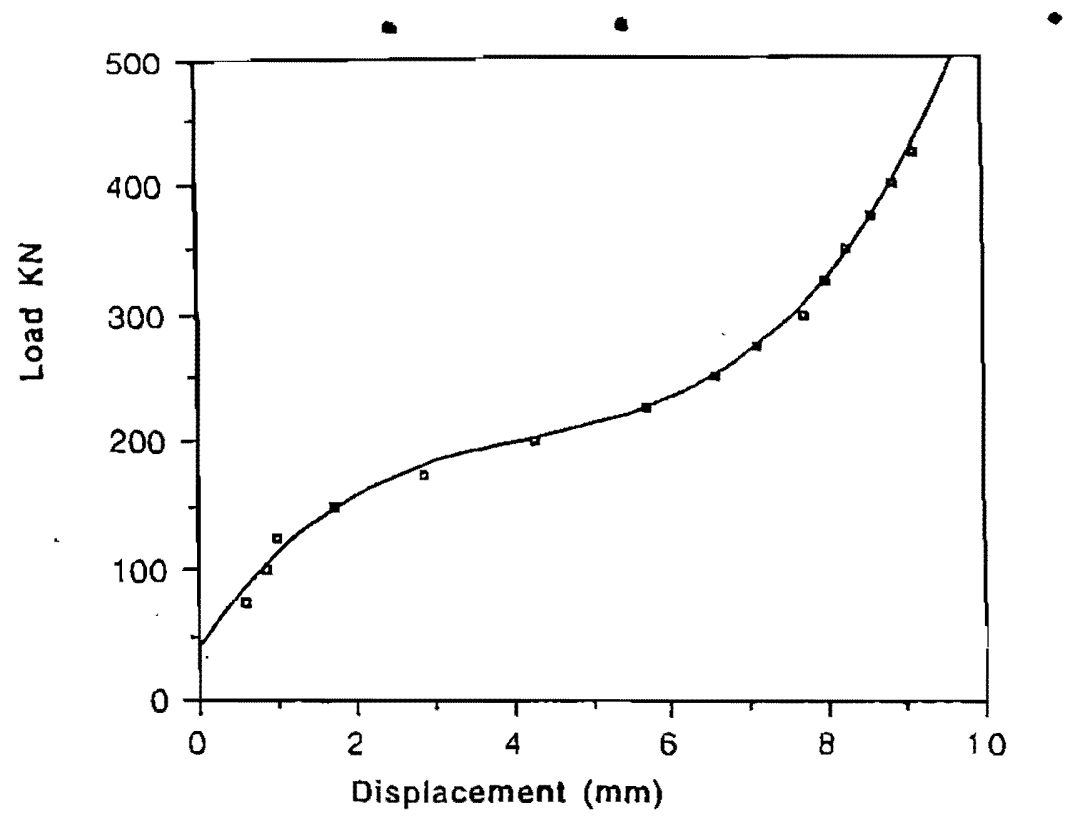

Fig. The experimental results of forging load versus displacement.

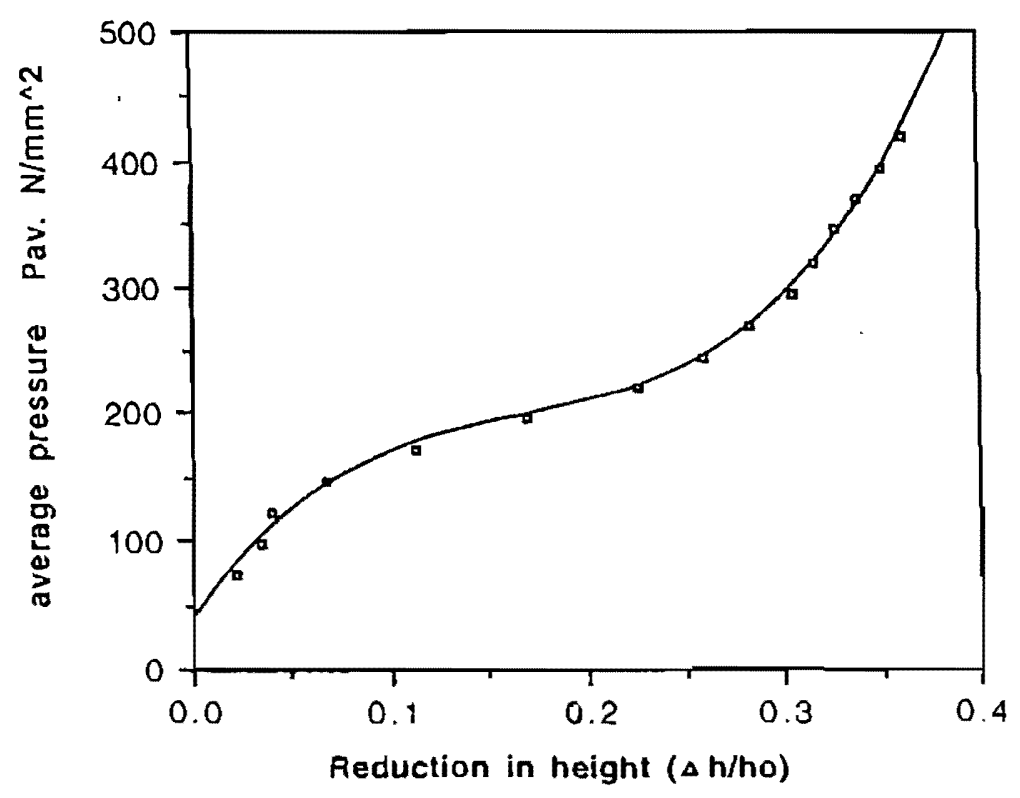

Fig. 9 The experimental average forging pressure versus reduction in height 


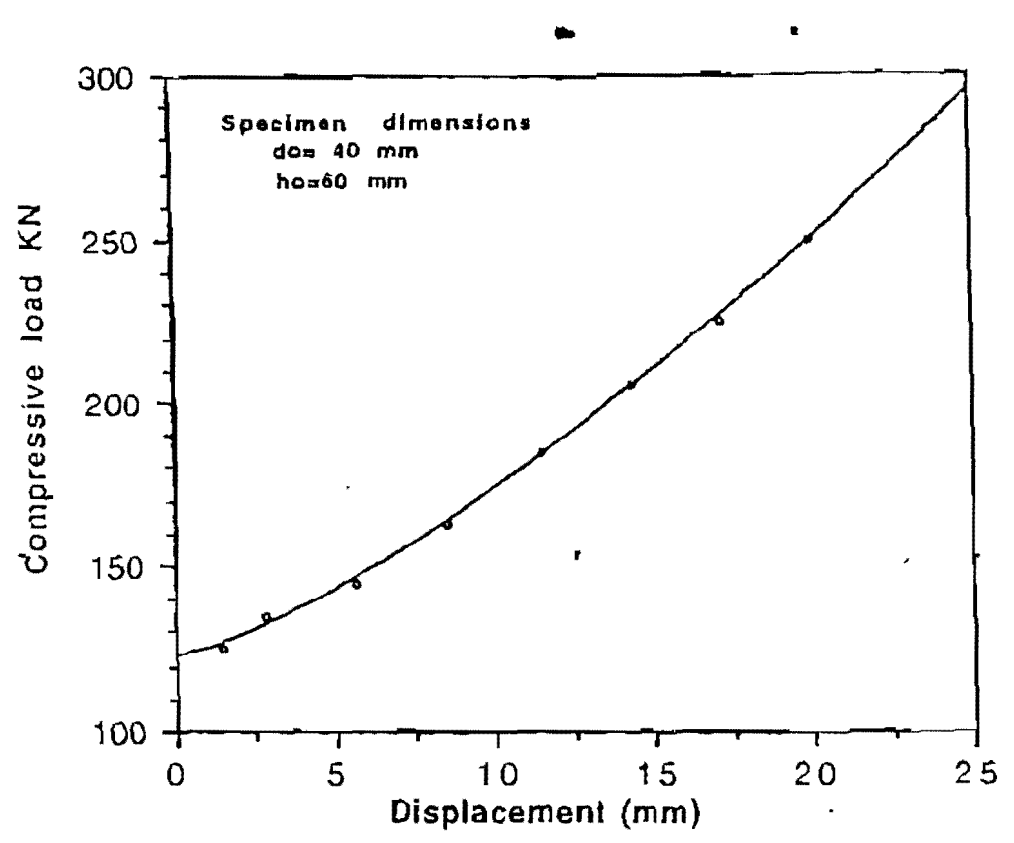

Fig.10 The experimental results of load versus displacement obtained tron Compression lest.

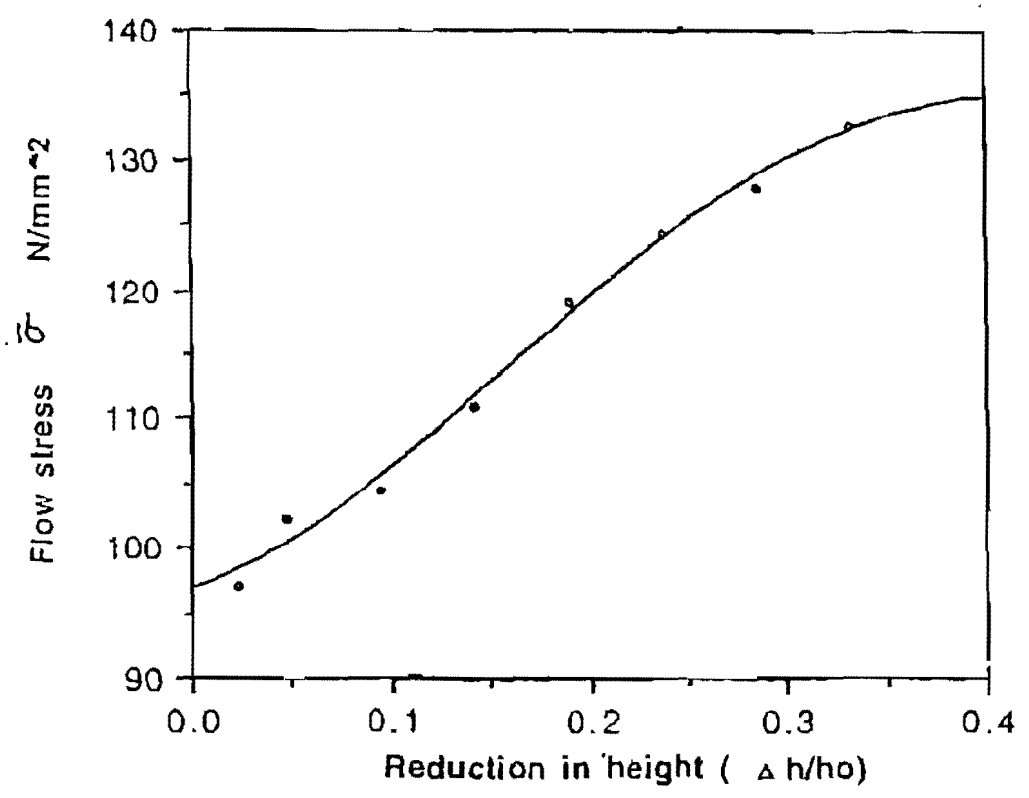

Fig.11 The experimental results of flow stress versus reduction in height for Compression lest. 


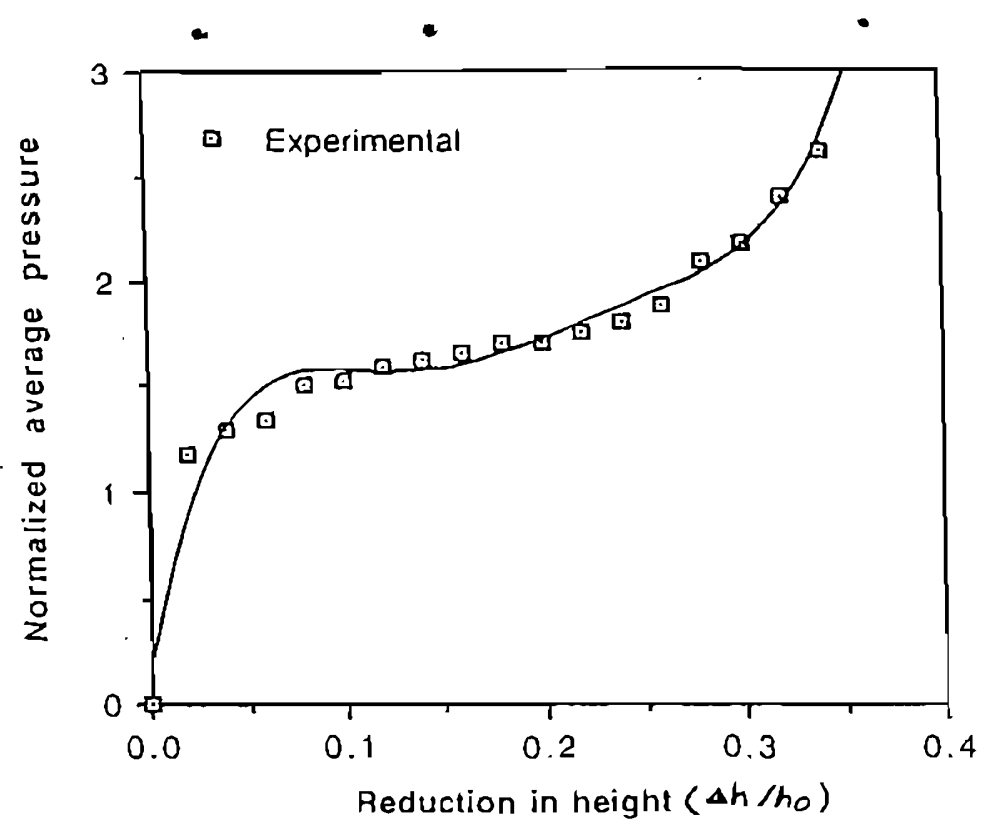

Fig.(12) The experimental results of normalized average pressure versus reduction in height

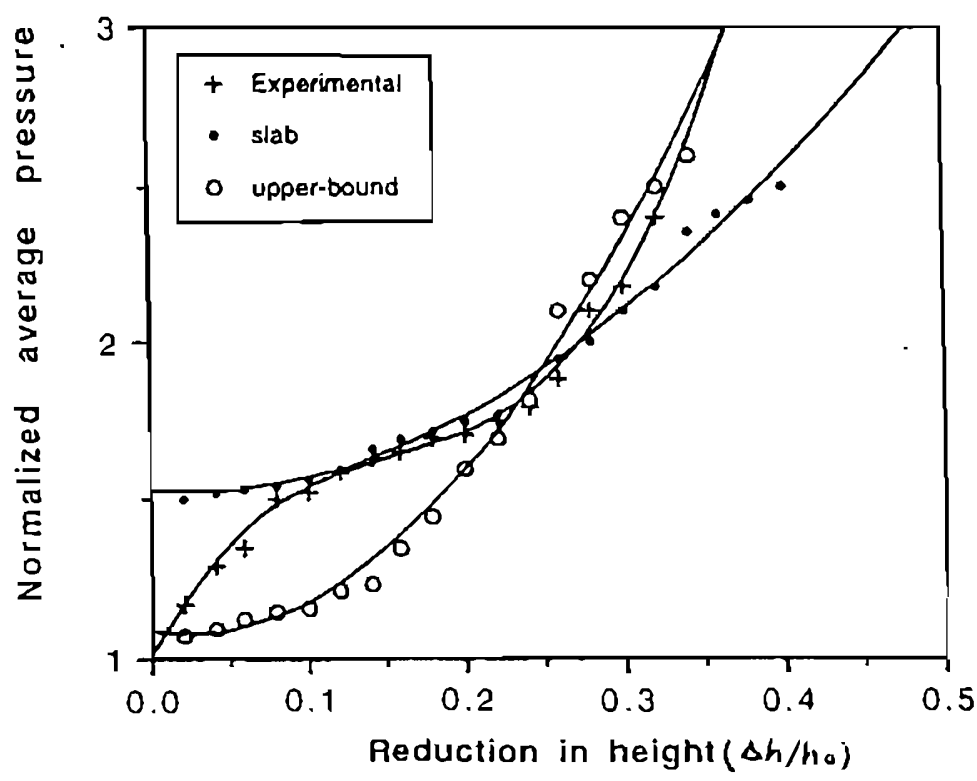

Fig.(13) Comparison between the experimental and the theorelical results 